\title{
MHD Convection Flow of Two Immiscible Fluids in an Inclined Channel with Heat Generation / Absorption
}

\author{
Hasan Nihal Zaidi ${ }^{1}$, Naseem Ahmad ${ }^{2}$ \\ ${ }^{1}$ Department of Basic Sciences, College of Preparatory Year, University of Hail, Hail, Kingdom of Saudi Arabia \\ ${ }^{2}$ Department of Mathematics, Jamia Millia Islamia, New Delhi, India
}

Email address:

hasannihalzaidi@hotmail.com (H. N. Zaidi),Naseem_mt@yahoo.com (N. Ahmad)

\section{To cite this article:}

Hasan Nihal Zaidi, Naseem Ahmad. MHD Convection Flow of Two Immiscible Fluids in an Inclined Channel with Heat Generation / Absorption. American Journal of Applied Mathematics. Vol. 4, No. 2, 2016, pp. 80-91. doi: 10.11648/j.ajam.20160402.13

Received: February 21, 2016; Accepted: March 5, 2016; Published: March 28, 2016

\begin{abstract}
Megnetohydrodynamic (MHD) convection flow of two immiscible fluids in an inclined channel in the presence of an applied magnetic field is investigated. Both fluids are assumed to be Newtonian and heat generating or absorbing and having constant transport properties. The channel walls are maintained at different temperature. The resulting coupled and nonlinear equations of momentum and energy are solved analytically by using the regular perturbation method valid for small value of $\varepsilon=\operatorname{PrEc}$. The influence of various parameters on velocity field and temperature field for heat absorption and heat generation are discussed with the aid of the graphs.
\end{abstract}

Keywords: MHD Convection, Two-Phase Flow/Immiscible Fluids, Heat Generation/Absorption, Regular Perturbation Method

\section{Introduction}

The convective flow and heat transfer of viscous incompressible electrically conducting fluid through a channel or pipe in the presence of a transverse magnetic field has important applications in magnetohydrodynamic (MHD) generator, pumps accelerators, flow meters, nuclear reactor and geothermal system. Hartmann [1] carried out the pioneer work on the study of steady MHD channel flow of a conducting fluid under a uniform magnetic field transverse to an electrically insulated channel. Later, effect of magnetic field on forced convection and heat transfer was studied by Seigal [2]. Osterle and Young [3], Perlmutter and Seigal [4], Romig [5] and Umavathi [6], investigated heat transfer in MHD flow between vertical parallel plates.

Many problems in the field of plasma physics, aeronautics, geophysics and petroleum industry involves multi layeredfluid flow. In the petroleum industry, as well as in other engineering and technological fields, a stratified two-phase fluid flow often occurs. For example, in geophysics, it is important to study the interaction of the geomagnetic field with the hot springs/fluids in geothermal regions. Once the interaction of the geomagnetic field with the flow field is known, then one can easily find the temperature distribution from the energy equation. Thome [7] initiated the first investigation associated with the two phase flow. Lohrasbi and Sahai [8], dealt with two-phase MHD flow and heat transfer in a parallel-plate channel. Malashetty and Leela [9, 10], have analyzed the Hartmann flow characteristics of twofluids in a horizontal channel. Chamkha [11] considered the steady, laminar flow of two viscous incompressible electrically conducting and heat generating or absorbing immiscible fluids in infinitely long porous and nonporous channels. Flow and Heat Transfer of Two Micropolar Fluids Separated by a Viscous Fluid Layer investigated by J. C. Umavathi, A. J. Chamkha, and M. Shekar [17].

In recent years, the various studies dealing with convective heat transfer in an inclined channel have been reported. The study showed that the tilting of the channel have significant effect on the flow and heat transfer characteristic. Prakash [12], investigated the liquid flowing in an open inclined channel. The viscous flow in an open inclined channel with naturally permeability bed was presented by Verma and Vyas [13]. Wang and Robillard [16], analyzed the mixed convection in an inclined channel with localized heat sources. A two-phase MHD flow and heat transfer in an inclined channel had been investigated by Malashetty et al. 
$[14,15]$. Double-Diffusive Natural Convection in Inclined Finned Triangular Porous Enclosures in the Presence of Heat Generation/Absorption Effects had been discussed by A.J. Chamkha et al [18].

None of the above mentioned authors considered the two phase MHD convective flow of electrically conducting fluid through an inclined channel with heat generation and absorption. This problem has been solved analytically for velocity as well as temperature distribution by using the regular perturbation method for small value of $\varepsilon(=\operatorname{Pr} E c)$. The influence of various dimensionless parameters on velocity and temperature field has been discussed graphically.

\section{Mathematical Formulation}

Consider steady, laminar, hydromagnetic and fully developed flow of two immiscible fluids through an inclined infinitely long channel. The channel walls are maintained at different temperatures $T_{w 1}$ and $T_{w 2}\left(T_{w 1}>T_{w 2}\right)$ extending in the $x-$ and $z$ - directions making an angle $\alpha$ with the horizontal. A uniform magnetic field of strength $B_{0}$ is applied transverse to the flow field. The regions $0 \leq y \leq h_{1}$ (Region-I) and $-h_{2} \leq y \leq 0$ (Region-II) are occupied by viscous incompressible and electrically conducting fluids. Both fluids are assumed to be Newtonian and heat generating or absorbing and having constant properties except the density in the buoyancy term of the momentum equation.

The governing equation of the motion and energy under the above stated assumptions can be written as

$$
\mu_{i} \frac{d^{2} u_{i}}{d y^{2}}+\rho_{i} g \beta_{i}\left(T_{i}-T_{w 2}\right) \sin \alpha-\sigma_{i} B_{0}^{2} u_{i}=\frac{\partial P}{\partial x}
$$

and

$$
K_{i} \frac{d^{2} T_{i}}{d y^{2}}+\mu_{i}\left(\frac{d u_{i}}{d y}\right)^{2}+\sigma_{i} B_{0}^{2} u_{i}^{2} \pm Q_{i}\left(T_{i}-T_{w 2}\right)=0,
$$

where $u_{i}$ and $T_{i}$ are the components of velocity and temperature respectively. $\rho_{i}, \mu_{i}, \sigma_{i}, K_{i}, \beta_{i}$ and $Q_{i}$ are density, viscosity, electrical conductivity, thermal conductivity, coefficient of thermal expansion and heat generation or absorption coefficient respectively.

We also assume that the fluids in both regions share a common pressure gradient $(\partial P / \partial x)$. The positive sign for $Q_{i}$ corresponds to heat generation and negative sign for heat absorption.

The boundary and interface conditions on velocity and temperature are

$$
\begin{gathered}
u_{1}\left(h_{1}\right)=0, \\
u_{1}(0)=u_{2}(0), \\
u_{2}\left(-h_{2}\right)=0, \\
\mu_{1} \frac{d u_{1}}{d y}=\mu_{2} \frac{d u_{2}}{d y} \text { at } y=0, \\
T_{1}\left(h_{1}\right)=T_{w 1}, \\
T_{1}(0)=T_{2}(0), \\
T_{2}\left(-h_{2}\right)=T_{w 2}
\end{gathered}
$$

and

$$
K_{1} \frac{d T_{1}}{d y}=K_{2} \frac{d T_{2}}{d y} \text { at } y=0
$$

The boundary conditions indicate the no slip and isothermal condition at the walls of channel. The fluid velocity, temperature, shear stress and heat flux are continuous across the interface.

Introducing the following dimensionless quantities in the equations (1) through (4) and

$$
\begin{gathered}
y_{i}^{\prime}=\frac{y_{i}}{h_{i}} ; u_{i}^{\prime}=\frac{u_{i}}{\bar{u}_{1}} ; \theta_{i}=\frac{T_{i}-T_{w 2}}{\Delta T} ; m=\frac{\mu_{1}}{\mu_{2}} ; k=\frac{K_{1}}{K_{2}} ; h=\frac{h_{2}}{h_{1}} ; n=\frac{\rho_{2}}{\rho_{1}} ; b=\frac{\beta_{2}}{\beta_{1}} ; \\
s=\frac{\sigma_{2}}{\sigma_{1}} ; G r=\frac{g \beta_{1} h_{1}^{3} \Delta T}{v_{1}^{2}} ; M=B_{0} \mathrm{~h}_{1} \sqrt{\frac{\sigma_{1}}{\mu_{1}}} ; P_{r}=\frac{\mu_{1} \mathrm{c}_{\mathrm{p}}}{\mathrm{K}_{1}} ; E_{c}=\frac{\overline{\mathrm{u}}_{1}^{2}}{\mathrm{c}_{\mathrm{p} \Delta \mathrm{T}}} ; R_{e}=\frac{\overline{\mathrm{u}}_{1} \mathrm{~h}_{1}}{v_{1}} ; P=\frac{h^{2}\left(\frac{\partial p}{\partial x}\right)}{\mu_{1} \bar{u}_{1}} ; \phi_{i}=\mathrm{Q}_{1} \frac{\mathrm{h}_{\mathrm{i}}^{2}}{\mathrm{k}_{\mathrm{i}}}
\end{gathered}
$$

suppresses the dash (/) for our convenience, we get

$$
\frac{d^{2} u_{i}}{d y^{2}}+\left(\frac{G r}{R e}\right) A \sin \alpha \theta_{i}-B M^{2} u_{i}=C P
$$

and

$$
\frac{d^{2} \theta_{i}}{d y^{2}}+\operatorname{Pr} E_{c} D\left(\frac{d u_{i}}{d y}\right)^{2}+\operatorname{Pr} E_{c} F M^{2} u_{i}^{2} \pm \phi_{i} \theta_{i}=0
$$

where $A=b m n h^{2}, B=m s h^{2}, C=m h^{2}, D=\frac{K}{m}$ and $F=$ $K h^{2} s . A, B, C, D$ and $F$ are equal to 1 for region- I.

The dimensionless boundary and interface conditions for velocity and temperature are:

$$
u_{1}(1)=0,
$$

$$
\begin{gathered}
u_{1}(0)=u_{2}(0), \\
u_{2}(-1)=0, \\
\frac{d u_{1}}{d y}=\left(\frac{1}{m h}\right) \frac{d u_{2}}{d y} \text { at } y=0, \\
\theta_{1}(1)=1 \\
\theta_{1}(0)=\theta_{2}(0), \\
\theta_{2}(-1)=0
\end{gathered}
$$

and

$$
\frac{d \theta_{1}}{d y}=\left(\frac{1}{k h}\right) \frac{d \theta_{2}}{d y} \text { at } y=0 .
$$




\section{Solution of the Problem}

The governing equations (5) and (6) are coupled and nonlinear because of buoyance force and the Ohmic dissipation term. In most of the practical problem the Eckert number is very small and hence the product $\epsilon=\operatorname{Pr} E_{c}$ can be treated as perturbation parameter to find the solution of equations (5) and (6) using regular perturbation method. The solutions are assumed in the form of

$$
\left(u_{i}, \theta_{i}\right)=\left(u_{i, 0}, \theta_{i, 0}\right)+\epsilon\left(u_{i, 1}, \theta_{i, 1}\right)+\ldots \ldots \ldots \ldots i=1,2 .(9)
$$

where $u_{i 0}, \theta_{i 0}$ are solutions for the case $\epsilon$ equal to zero. The quantities $u_{i 1}$ and $\theta_{i 1}$ are corrections relating to $u_{i 0}$ and $\theta_{i 0}$ respectively.

Substituting the equation (9) in the equations (5) and (6) and equating coefficients of the like powers of $\epsilon$ to zero, we get the following set of equations:

Zeroth order

$$
\frac{d^{2} u_{i, 0}}{d y^{2}}+\left(\frac{G r}{R e}\right) A \sin \alpha \theta_{i, 0}-B M^{2} u_{i, 0}=C P
$$

and

$$
\frac{d^{2} \theta_{i, 0}}{d y^{2}} \pm \phi_{i} \theta_{i, 0}=0
$$

First order

$$
\frac{d^{2} u_{i, 1}}{d y^{2}}+\left(\frac{G r}{R e}\right) A \sin \alpha \theta_{i, 1}-B M^{2} u_{i, 1}=0
$$

and

$$
\frac{d^{2} \theta_{i, 1}}{d y^{2}}+D\left(\frac{d u_{i, 0}}{d y}\right)^{2}+F M^{2} u_{i, 0}^{2} \pm \phi_{i} \theta_{i, 1}=0
$$

The corresponding boundary and interface conditions 7(ad) and 8(a-d) using equation (9) become

Zeroth order

$$
\begin{aligned}
& u_{1,0}(1)=0, \\
& u_{1,0}(0)=u_{2,0}(0), \\
& u_{2,0}(-1)=0,
\end{aligned}
$$

$$
\begin{aligned}
& \frac{d u_{1,0}}{d y}=\left(\frac{1}{m h}\right) \frac{d u_{2,0}}{d y} \text { at } y=0 \\
& \theta_{1,0}(1)=1 \\
& \theta_{1,0}(0)=\theta_{2,0}(0) \\
& \theta_{2,0}(-1)=0
\end{aligned}
$$

and

$$
\frac{d \theta_{1,0}}{d y}=\left(\frac{1}{k h}\right) \frac{d \theta_{2,0}}{d y} \text { at } y=0
$$

First order

$$
\begin{gathered}
u_{1,1}(1)=0, \\
u_{1,1}(0)=u_{2,1}(0), \\
u_{2,1}(-1)=0, \\
\frac{d u_{1,1}}{d y}=\left(\frac{1}{m h}\right) \frac{d u_{2,1}}{d y} \text { at } y=0, \\
\theta_{1,1}(1)=0, \\
\theta_{1,1}(0)=\theta_{2,1}(0), \\
\theta_{2,1}(-1)=0
\end{gathered}
$$

and

$$
\frac{d \theta_{1,1}}{d y}=\left(\frac{1}{k h}\right) \frac{d \theta_{2,1}}{d y} \text { at } y=0
$$

There are two different solutions for temperature fields corresponding to the case of heat absorption and heat generation fluids.

Case I: Heat absorption

The solutions of Equations (10), (11), (12) and (13) by using the boundary conditions 14(a-d), 15(a-d), 16(a-d) and $17(\mathrm{a}-\mathrm{d})$ with negative sign for $\phi_{i}$ are given as follows:

Region-I

Zeroth order

$$
\theta_{1,0}=c_{1} \cosh \left(\sqrt{\phi_{1}} y\right)+c_{2} \sinh \left(\sqrt{\phi_{1}} y\right)
$$

First order

$$
\begin{array}{r}
\theta_{1,1}=c_{9} \cosh \left(\sqrt{\phi_{1}} y\right)+c_{10} \sinh \left(\sqrt{\phi_{1}} y\right)-B_{7} \cosh \left(2 \sqrt{\phi_{1}} y\right)-B_{8} \sinh (2 M y)-B_{9} \cosh (M y)+B_{10} \sinh (M y)+ \\
B_{11} \sinh \left(B_{3} y\right)+B_{12} \sinh \left(B_{4} y\right)+B_{13} \cosh \left(B_{3} y\right)+B_{14} \cosh \left(B_{4} y\right)+B_{15} y \cdot \cosh \left(\sqrt{\phi_{1}} y\right)-B_{16} \sinh \left(2 \sqrt{\phi_{1}} y\right)- \\
B_{17} y \cdot \sinh \left(\sqrt{\phi_{1}} y\right)+B_{18} \\
(20) \\
u_{1,1}=c_{13} \cosh (M y)+c_{14} \sinh (M y)+\left(l_{4}-l_{18}\right) \cosh \left(\sqrt{\phi_{1}} y\right)+\left(l_{5}+l_{15}\right) \sinh \left(\sqrt{\phi_{1}} y\right)+l_{6} \cosh \left(2 \sqrt{\phi_{1}} y\right)+ \\
l_{7} \sinh (2 M y)+l_{8} y \sinh (M y)+l_{9} y \cosh (M y)-l_{10} \sinh \left(B_{3} y\right)-l_{11} \sinh \left(B_{4} y\right)-l_{12} \cosh \left(B_{3} y\right)-l_{13} \cosh \left(B_{4} y\right)+ \\
l_{14} y \cosh \left(\sqrt{\phi_{1}} y\right)+l_{16} \sinh \left(2 \sqrt{\phi_{1}} y\right)-l_{17} y \sinh \left(\sqrt{\phi_{1}} y\right)+l_{19}
\end{array}
$$




$$
\begin{gathered}
\theta_{2,0}=c_{3} \cosh \left(\sqrt{\phi_{2}} y\right)+c_{4} \sinh \left(\sqrt{\phi_{2}} y\right) \\
u_{2,0}=c_{7} \cosh (M \sqrt{B} y)+c_{8} \sinh (M \sqrt{B} y)-\frac{C P}{B M^{2}}-A_{5}\left[c_{3} \cosh \left(\sqrt{\phi_{2}} y\right)+c_{4} \sinh \left(\sqrt{\phi_{2}} y\right)\right]
\end{gathered}
$$

First order

$$
\begin{gathered}
\theta_{2,1}=c_{11} \cosh \left(\sqrt{\phi_{2}} y\right)+c_{12} \sinh \left(\sqrt{\phi_{2}} y\right)-G_{7} \cosh (2 M \sqrt{B} y)-G_{8} \sinh (2 M \sqrt{B} y)-G_{9} \cosh \left(G_{2} y\right)+G_{10} \cosh \left(G_{3} y\right)+ \\
G_{11} \sinh \left(G_{2} y\right)-G_{12} \sinh \left(G_{3} y\right)-G_{13} \sinh \left(2 \sqrt{\phi_{2}} y\right)-G_{14} \cosh \left(2 \sqrt{\phi_{2}} y\right)+G_{15} \cosh \left(B_{1} y\right)+G_{16} \sinh \left(B_{1} y\right)- \\
G_{17} y \cdot \sinh \left(\sqrt{\phi_{2}} y\right)+G_{18} y \cdot \cosh \left(\sqrt{\phi_{2}} y\right)+G_{19}
\end{gathered}
$$

$u_{2,1}=c_{15} \cosh (M \sqrt{B} y)+c_{16} \sinh (M \sqrt{B} y)+\left(m_{4}-m_{17}\right) \cosh \left(\sqrt{\phi_{2}} y\right)+\left(m_{5}+m_{19}\right) \sinh \left(\sqrt{\phi_{2}} y\right)$

$+m_{6} \cosh (2 M \sqrt{B} y)+m_{7} \sinh (2 M \sqrt{B} y)+m_{8} \cosh \left(G_{3} y\right)-m_{9} \cosh \left(G_{4} y\right)-m_{10} \sinh \left(G_{3} y\right)+m_{11} \sinh \left(G_{4} y\right)$

$+m_{12} \sinh \left(2 \sqrt{\phi_{2}} y\right)+m_{13} \cosh \left(2 \sqrt{\phi_{2}} y\right)-m_{14} y \sinh (M \sqrt{B} y)+m_{15} y \cosh (M \sqrt{B} y)$

$$
-m_{16} y \sinh \left(\sqrt{\phi_{2}} y\right)+m_{18} y \cosh \left(\sqrt{\phi_{2}} y\right)+m_{20}
$$

\section{Case II: Heat Generation}

For the heat generation case, the equations (10), (11), (12) and (13) with boundary conditions 14(a-d), 15(a-d), 16(a-d) and $17(\mathrm{a}-\mathrm{d})$ are solved for positive sign of $\phi_{i}$. The solutions are given as follows:

Region- I

Zeroth order

$$
\begin{gathered}
\theta_{1,0}=d_{1} \sin \left(\sqrt{\phi_{1}} y\right)+d_{2} \cos \left(\sqrt{\phi_{1}} y\right) \\
u_{1,0}=d_{5} e^{M y}+d_{6} e^{-M y}-\frac{P}{M^{2}}+D_{2}\left\{d_{1} \sin \left(\sqrt{\phi_{1}} y\right)+d_{2} \cos \left(\sqrt{\phi_{1}} y\right)\right\}
\end{gathered}
$$

First order

$$
\begin{gathered}
\theta_{1,1}=d_{9} \sin \left(\sqrt{\phi_{1}} y\right)+d_{10} \cos \left(\sqrt{\phi_{1}} y\right)-a_{3} e^{2 M y}-a_{4} e^{-2 M y}+a_{5} \cos \left(2 \sqrt{\phi_{1}} y\right)-2 a_{6} e^{M y} \cos \left(\sqrt{\phi_{1}} y\right) \\
-2 a_{7} e^{M y} \sin \left(\sqrt{\phi_{1}} y\right)-2 a_{8} e^{-M y} \sin \left(\sqrt{\phi_{1}} y\right)-2 a_{9} e^{-M y} \cos \left(\sqrt{\phi_{1}} y\right)+a_{10} \sin \left(2 \sqrt{\phi_{1}} y\right)+a_{11} e^{M y}+a_{12} e^{-M y} \\
+a_{13} y \cos \left(\sqrt{\phi_{1}} y\right)-a_{14} y \sin \left(\sqrt{\phi_{1}} y\right)-a_{15}
\end{gathered}
$$

$$
\begin{array}{r}
u_{1,1}=d_{13} e^{M y}+d_{14} e^{-M y}+n_{1} \sin \left(\sqrt{\phi_{1}} y\right)+n_{2} \cos \left(\sqrt{\phi_{1}} y\right)+n_{3} e^{2 M y}+n_{4} e^{-2 M y}+n_{5} \cos \left(2 \sqrt{\phi_{1}} y\right)+n_{6} \sin \left(2 \sqrt{\phi_{1}} y\right)- \\
n_{7} y e^{M y}+n_{8} y e^{-M y}+n_{9} e^{M y} \sin \left(\sqrt{\phi_{1}} y\right)-n_{10} e^{M y} \cos \left(\sqrt{\phi_{1}} y\right)-n_{11} e^{-M y} \sin \left(\sqrt{\phi_{1}} y\right)+n_{12} e^{-M y} \cos \left(\sqrt{\phi_{1}} y\right)+ \\
n_{13} y \cos \left(\sqrt{\phi_{1}} y\right)-n_{14} \sin \left(\sqrt{\phi_{1}} y\right)-n_{15} y \sin \left(\sqrt{\phi_{1}} y\right)-n_{16} \cos \left(\sqrt{\phi_{1}} y\right)-n_{17}
\end{array}
$$

Region- II

Zeroth order

$$
\begin{gathered}
\theta_{2,0}=d_{3} \sin \left(\sqrt{\phi_{2}} y\right)+d_{4} \cos \left(\sqrt{\phi_{2}} y\right) \\
u_{2,0}=d_{7} e^{M \sqrt{B} y}+d_{8} e^{-M \sqrt{B} y}-\frac{P}{B M^{2}}+D_{3}\left\{d_{3} \sin \left(\sqrt{\phi_{2}} y\right)+d_{4} \cos \left(\sqrt{\phi_{2}} y\right)\right\}
\end{gathered}
$$

First order

$$
\begin{gathered}
\theta_{2,1}=d_{11} \sin \left(\sqrt{\phi_{2}} y\right)+d_{12} \cos \left(\sqrt{\phi_{2}} y\right)-g_{4} e^{2 M \sqrt{B} y}+g_{5} e^{-2 M \sqrt{B} y}-g_{6} \cos \left(2 \sqrt{\phi_{2}} y\right)-g_{7} e^{M \sqrt{B} y} \sin \left(\sqrt{\phi_{2}} y\right)- \\
g_{8} e^{M \sqrt{B} y} \cos \left(\sqrt{\phi_{2}} y\right)-g_{9} e^{-M \sqrt{B} y} \cos \left(\sqrt{\phi_{2}} y\right)+g_{10} e^{-M \sqrt{B} y} \sin \left(\sqrt{\phi_{2}} y\right)+g_{11} \sin \left(2 \sqrt{\phi_{2}} y\right)- \\
g_{12} y \cos \left(\sqrt{\phi_{2}} y\right)+g_{13} y \sin \left(\sqrt{\phi_{2}} y\right)+g_{14} e^{M \sqrt{B} y}+g_{15} e^{-M \sqrt{B} y}-g_{16} \\
p_{6} \sin \left(2 \sqrt{\phi_{2}} y\right)-b_{7} y e^{M \sqrt{B} y}+p_{8} y e^{-M \sqrt{B} y}+p_{9} e^{M \sqrt{B} y} \sin \left(\sqrt{\phi_{2}} y\right)-p_{10} e^{M \sqrt{B} y} \cos \left(\sqrt{\phi_{2}} y\right)-p_{11} e^{-M \sqrt{B} y} \sin \left(\sqrt{\phi_{2}} y\right)- \\
p_{12} e^{-M \sqrt{B} y} \cos \left(\sqrt{\phi_{2}} y\right)-p_{13} y \cos \left(\sqrt{\phi_{2}} y\right)-p_{14} \sin \left(\sqrt{\phi_{2}} y\right)+p_{15} y \sin \left(\sqrt{\phi_{2}} y\right)+p_{16} \cos \left(\sqrt{\phi_{2}} y\right)-p_{17}
\end{gathered}
$$

It should be noted that the constants appearing in the equations (18)-(33) are defined in the Appendix section, since the problem involves large number of dimensionless parameters. However, for simplicity we fix some of them.
The values for the parameters $(P, \operatorname{Re}, G r, \alpha, k, n, b)$ are fixed as $(-5,5,1, \pi / 6,1,2,2)$ for all the numerical computations. 


\section{Results and Discussion}

In this paper, we have analysed MHD convection flow and heat transfer for two immiscible fluids in an inclined channel with heat absorption and heat generation. We draw the following conclusions on the basis of graphs, which have been drawn to judge the variation of velocity and temperature fields with respect to the various parameters. We summarize the result in the following paragraphs.

Fig. 1 shows the variation of velocity for different values of the height ratio $h$ for the case of heat absorption. It is observed that the velocity of fluid increases with the increase of $h$. For the large value of $h$, the velocity profile for both regions is almost same at the interface line $y=0$.

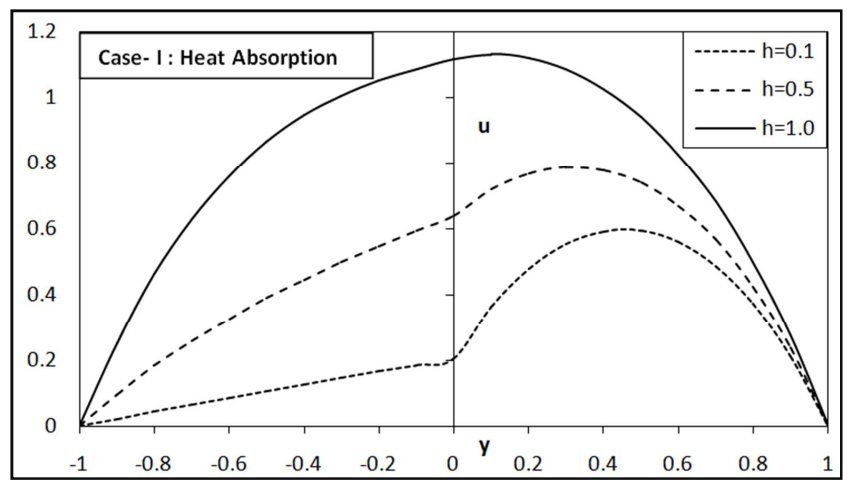

Fig. 1. Velocity profile for different values of the height ratio $h$.

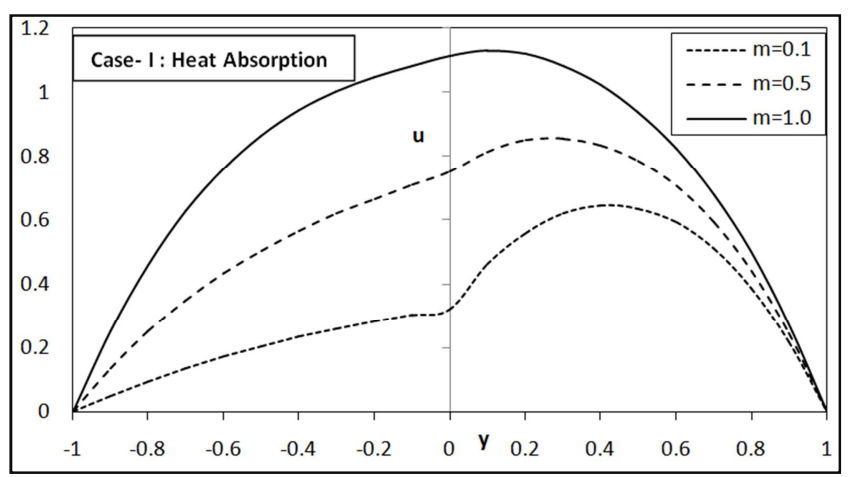

Fig. 2. Velocity profile for different values of the viscosity ratio $m$.

Fig. 2 shows the variation of velocity for different values of the viscosity ratio $m$. As the value of $m$ increases, the velocity of the fluid increases in the both regions. It is observed that the velocity of the fluid in the region I is smaller as compared to the velocity of the fluid in the region II.

The variation of the velocity profile with respect to the Grashof number $G r$ is shown in fig. 3. It is noticed that as the value of Grashof number $\mathrm{Gr}$ increases, the velocity of both fluids increases as expected. An increase in the Grashof number $G r$ physically means increase of the buoyancy force which supports the flow.

Fig. 4 exhibits that as Hartmann number $M$ increases, velocity field decreases. This is because the magnetic field slows down the velocity of fluid particles. This is classical Hartmann effect. The influence of heat absorption coefficient on the velocity profile is shown in the fig. 5. The heat absorption coefficient $\phi$ measures the amount of heat flux absorbed by the fluid particles. It is shown that the velocity of fluid particles decreases as the heat of absorption coefficient increases.

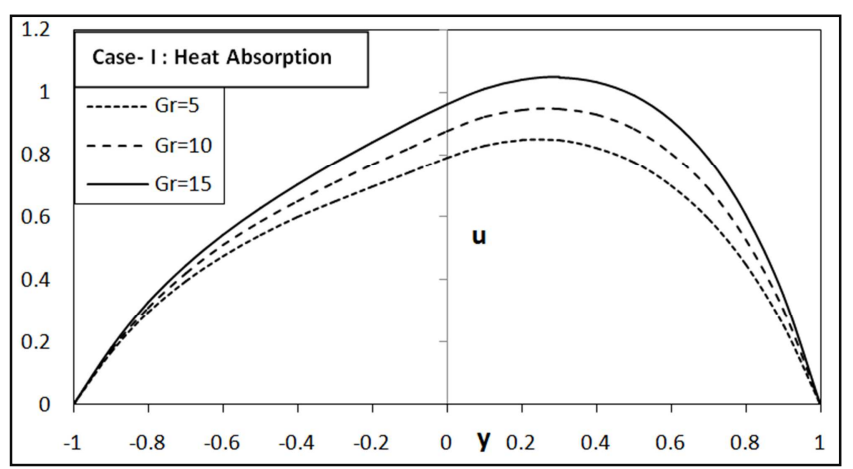

Fig. 3. Velocity profile for different values of the Grashof number Gr.

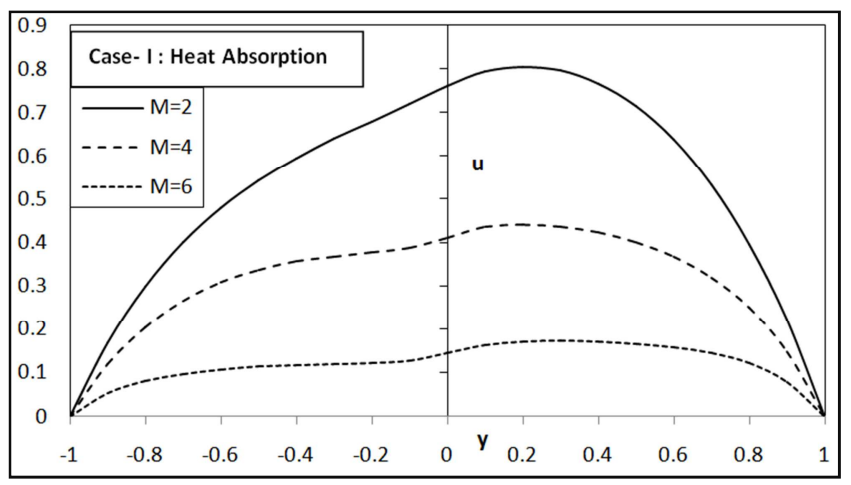

Fig. 4. Velocity profile for different values of the Hartmann number M.

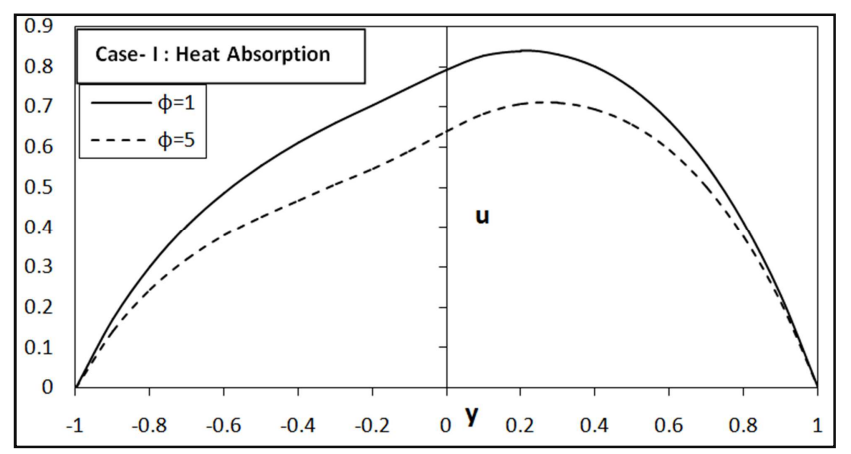

Fig. 5. Velocity profile for different value of heat absorption coefficient $\phi$.

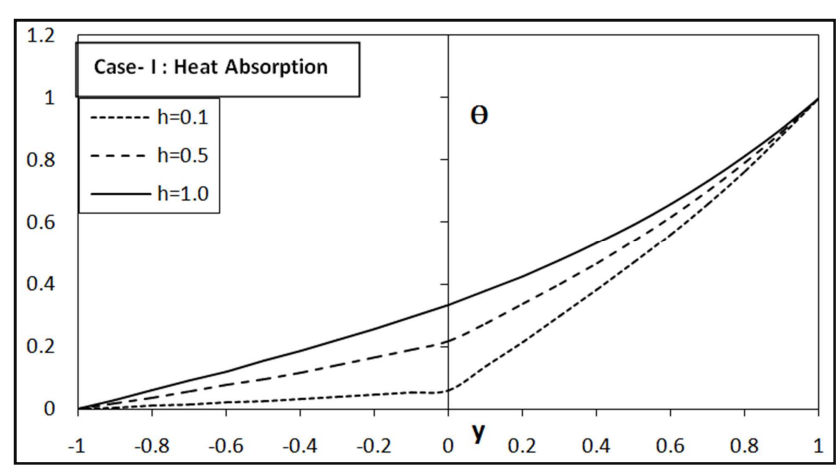

Fig. 6. Temperature profile for different values of the height ratio $h$. 
Fig. 6 shows the variation of temperature field with respect to the height ratio $h$. It is observed that an increase in the value of $h$ increases the temperature field. It is also noticed that the temperature profiles are linear for the smaller values of $h$, which indicates that the heat transfer is essential for conduction alone.

Fig. 7 shows the influence of the thermal conductivity ratio $k$ on the temperature profile. It is found that the temperature field increases with the increase in the value of $k$. The effect of heat absorption coefficient $\phi$ on temperature profile is shown in the fig. 8. It is clear from the figure that the temperature decreases as the value of $\phi$ increases.

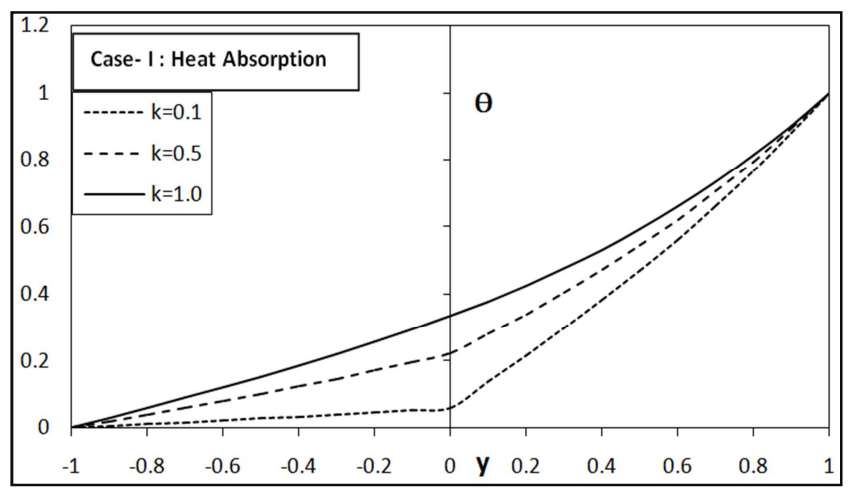

Fig. 7. Temperature profile for different values of thermal conductivity ratio $k$.

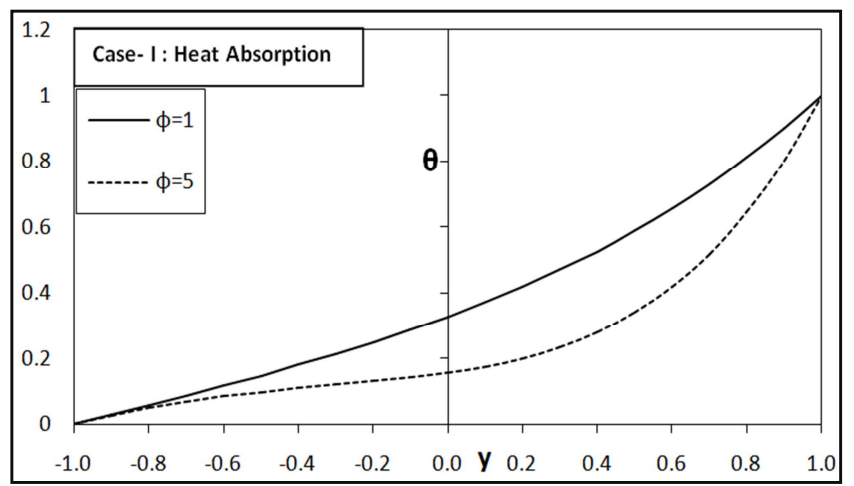

Fig. 8. Temperature profile for different values of the heat absorption coefficient $\phi$.

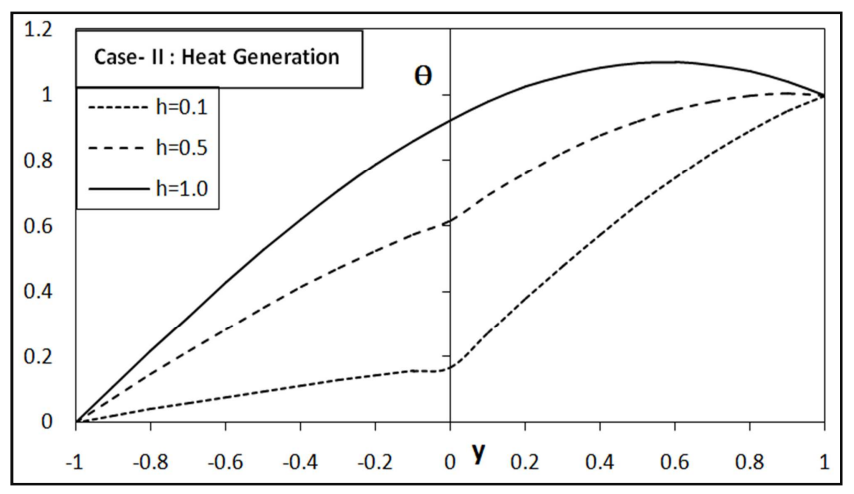

Fig. 9. Temperature profile for different values of the height ratio $h$.

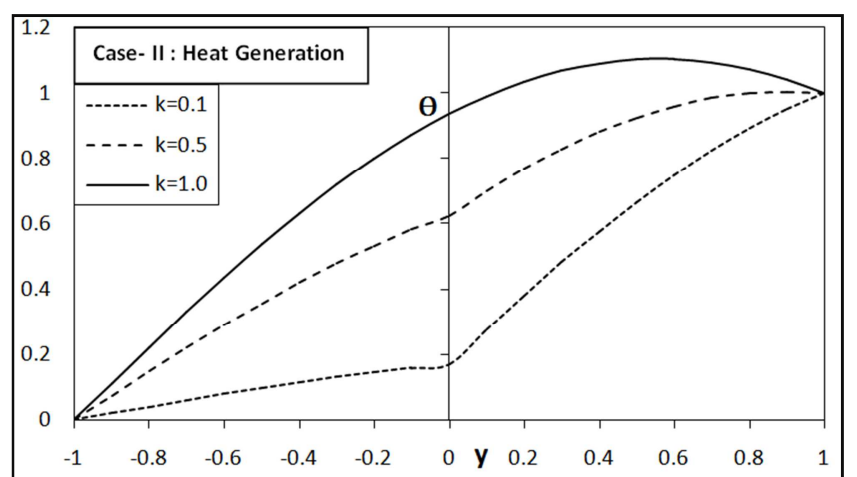

Fig. 10. Temperature profile for different values of the thermal conductivity ratio $k$.

Fig. 9 and fig. 10 display the effect of the height ratio $h$ and the thermal conductivity ratio $k$ on the temperature distribution in the case of heat generation. It is observed that there is an increase in the temperature field when the values of the height ratio $h$ and thermal conductivity ratio $k$ increases. It has also been noticed that the magnitude of temperature profile is larger in region II as compared to the magnitude of temperature profile in region $\mathrm{I}$. The temperature profiles for the case of heat generation are nonlinear indicates that the convection heat transfer is prominent.

Fig. 11 shows the effect of the angle of inclination $\alpha$ on the velocity profile. It is observed that velocity increases as angle of inclination increases. It happens due to the increase in the buoyancy force with inclination angle $\alpha$.

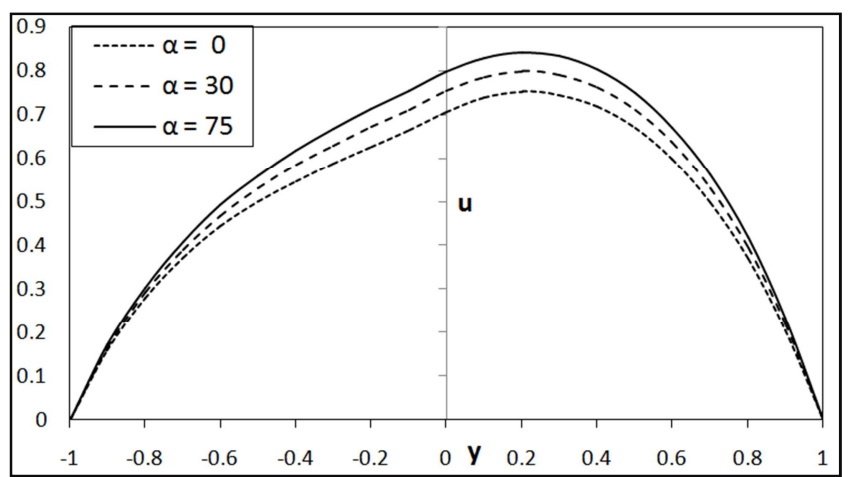

Fig. 11. Velocity profile for different values of angle of inclination $\alpha$.

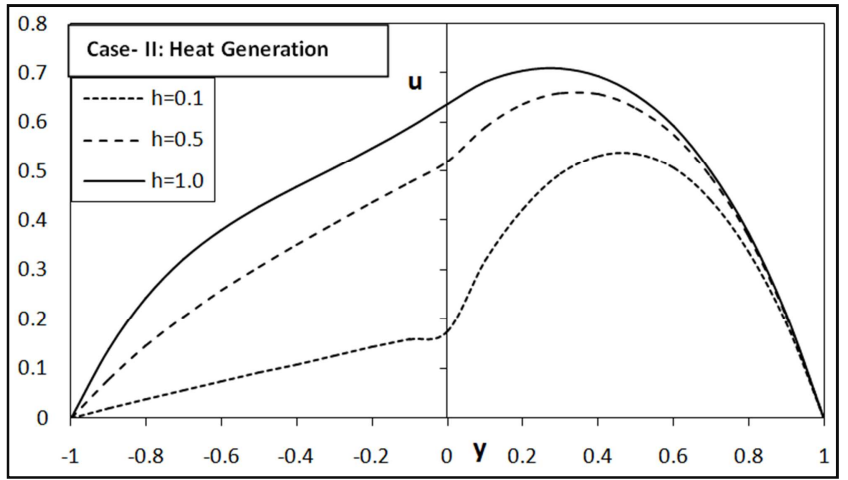

Fig. 12. Velocity profile for different values of the height ratio $h$. 


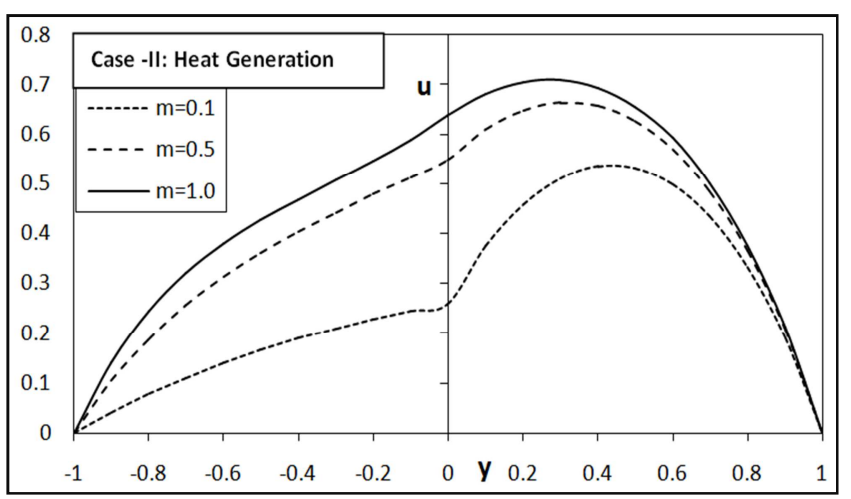

Fig. 13. Velocity profile for different values of the viscosity ratio $m$.

Fig. 12 and fig. 13 depict the effect of the height ratio $h$ and viscosity ratio $m$ on velocity profile in case of heat generation. It is evident that the velocity profile increases with the increase of the height ratio $h$ and the viscosity ratio $m$, but the magnitude of velocity is large in the region II.

Fig. 14 and fig. 15 show the variation of velocity profile for different values of Grashof number Gr and Hartmann number $\mathrm{M}$ respectively. The velocity profile increases with the increase of Grashof number while it is decreases with the increase of Hartmann number M. Fig. 16 shows the variation of velocity profile for different values of heat generation coefficient. It is evident that the velocity of the fluid particles increases with an increase of the heat generation coefficient $\phi$.

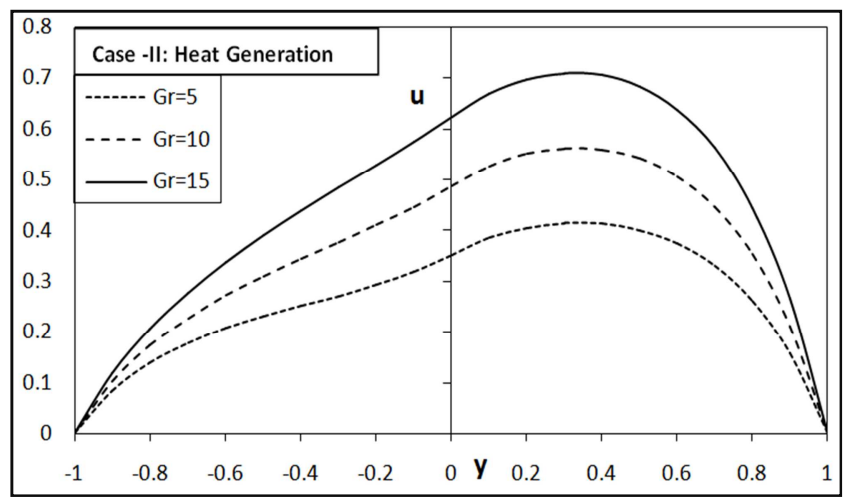

Fig. 14. Velocity profile for different values of the Grashof number Gr.

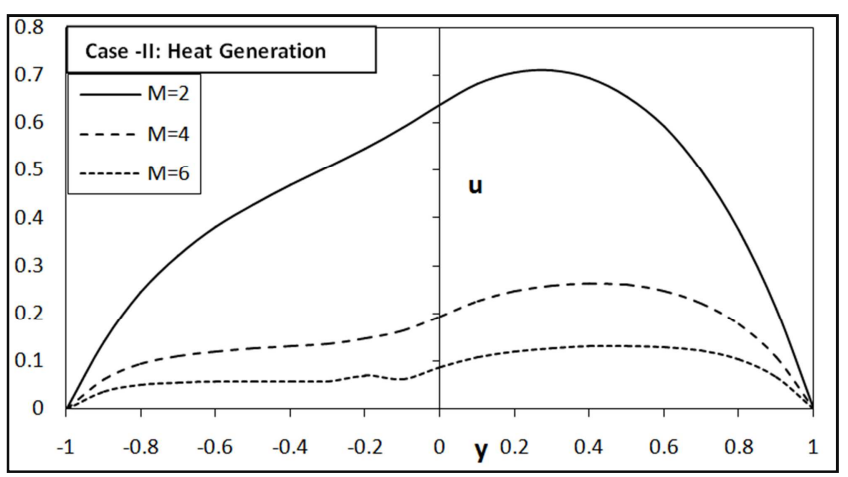

Fig. 15. Velocity profile for different values of the Hartmann number $M$.

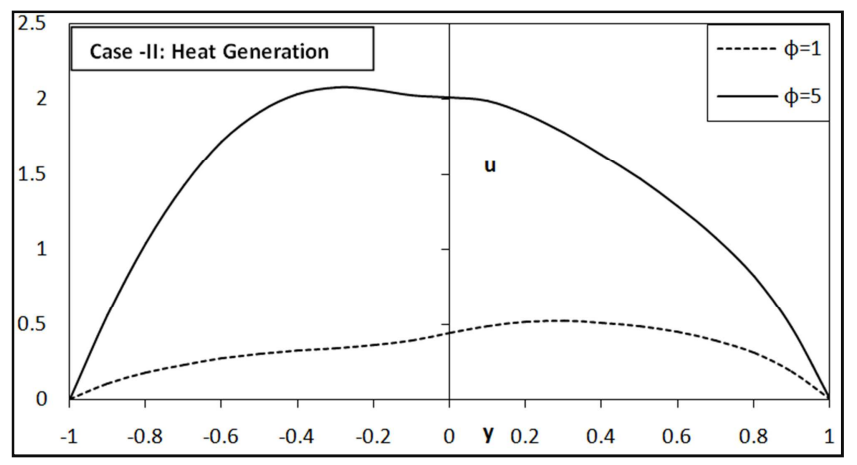

Fig. 16. Velocity profile for different values of heat generation coefficient $\phi$.

\section{Conclusion}

The problem of MHD convection flow of two immiscible fluids in an inclined channel is analyzed in the presence of magnetic field with heat generation and heat absorption. The governing coupled equations are solved analytically by using perturbation method for different values of the parameters. The main conclusions of the present analysis are as follows:

1. The height ratio, viscosity ratio, Grashof number, angle of inclination and heat generation coefficient promote the velocity field.

2. An increase in Hartmann number and heat absorption coefficient suppress the velocity and temperature profile.

3. An increase in the height ratio, thermal conductivity, Grahof number and heat generation coefficient increase the temperature field.

\section{Appendix}

$$
\begin{gathered}
A_{1}=k h \sqrt{\frac{\phi_{1}}{\phi_{2}}}, A_{2}=\sinh \left(\sqrt{\phi_{1}}\right) \cosh \left(\sqrt{\phi_{2}}\right)+A_{1} \cosh \left(\sqrt{\phi_{1}}\right) \sinh \left(\sqrt{\phi_{2}}\right), A_{3}=\frac{\mathrm{Gr}}{\mathrm{Re}} \cdot \sin \alpha \\
A_{4}=\frac{A_{3}}{\phi_{1}-M^{2}}, A_{5}=\frac{A \cdot A_{3}}{\phi_{1}-B M^{2}}, A_{6}=\frac{(B-C) P}{B M^{2}}-c_{1}\left(A_{4}+A A_{5}\right), A_{7}=\frac{A_{4} c_{2} \sqrt{\phi_{1}}-A A_{5} c_{4}\left(\frac{\sqrt{\phi_{2}}}{m h}\right)}{M} \\
\mathrm{~A}_{8}=\frac{\mathrm{P}}{\mathrm{M}^{2}}+\mathrm{A}_{4}\left\{\mathrm{c}_{1} \cosh \left(\sqrt{\phi_{1}}\right)-\mathrm{c}_{2} \sinh \left(\sqrt{\phi_{1}}\right)\right\}+\mathrm{A}_{7} \sinh (\mathrm{M}) \\
\mathrm{A}_{9}=\frac{\mathrm{CP}}{\mathrm{BM}^{2}}+\mathrm{A} \mathrm{A}_{5}\left\{\mathrm{c}_{3} \cosh \left(\sqrt{\phi_{2}}\right)-\mathrm{c}_{4} \sinh \left(\sqrt{\phi_{2}}\right)\right\}+\mathrm{A}_{6} \cosh (\mathrm{M} \sqrt{\mathrm{B}}) \\
\mathrm{A}_{10}=\mathrm{B}_{1} \sinh (\mathrm{M}) \cosh (\mathrm{M} \sqrt{\mathrm{B}})+\cosh (\mathrm{M}) \sinh (\mathrm{M} \sqrt{\mathrm{B}})
\end{gathered}
$$




$$
\begin{aligned}
& B_{1}=\frac{1}{M^{2}-\phi_{1}}, B_{2}=\frac{1}{4 M^{2}-\phi_{1}}, B_{3}=\sqrt{\phi_{1}}+M, B_{4}=\sqrt{\phi_{1}}-M, B_{5}=\frac{1}{B_{3}^{2}-\phi_{1}}, B_{6}=\frac{1}{B_{4}^{2}-\phi_{1}} \\
& B_{7}=\frac{A_{4}^{2}\left(c_{1}^{2}-c_{2}^{2}\right)}{6}\left(\frac{M^{2}}{\phi_{1}}-1\right), B_{8}=2 M^{2} c_{5} c_{6} B_{2}, B_{9}=2 P c_{5} B_{1}, B_{10}=2 P c_{6} B_{1} \\
& B_{11}=M A_{4} B_{6} B_{3}\left(c_{1} c_{6}+c_{2} c_{5}\right), B_{12}=M A_{4} B_{4} B_{5}\left(c_{1} c_{6}-c_{2} c_{5}\right), B_{13}=M A_{4} B_{6} B_{4}\left(c_{2} c_{6}-c_{1} c_{5}\right) \\
& B_{14}=M A_{4} B_{3} B_{5}\left(c_{1} c_{5}+c_{2} c_{6}\right), B_{15}=\frac{P A_{4} c_{2}}{\sqrt{\phi_{1}}}, B_{16}=\frac{A_{4}^{2} c_{1} c_{2}}{3}\left(\frac{M^{2}}{\phi_{1}}+1\right), B_{17}=\frac{P A_{4} c_{1}}{\sqrt{\phi_{1}}} \\
& B_{18}=\frac{P^{2}}{M^{4} \phi_{1}}+\frac{M^{2}}{\phi_{1}}\left(c_{5}^{2}+c_{6}^{2}\right)+\frac{A_{4}^{2}}{2}\left(c_{1}^{2}+c_{2}^{2}\right)\left(\frac{M^{2}}{\phi_{1}}+1\right) \\
& D_{1}=\sin \left(\sqrt{\phi_{1}}\right) \cos \left(\sqrt{\phi_{2}}\right)+A_{1} \cos \left(\sqrt{\phi_{1}}\right) \sin \left(\sqrt{\phi_{2}}\right), D_{2}=\frac{A_{3}}{\phi_{1}+M^{2}}, D_{3}=\frac{A \cdot A_{3}}{\phi_{1}+B M^{2}} \\
& D_{4}=\frac{P}{M^{2}}-D_{2}\left\{d_{1} \sin \left(\sqrt{\phi_{1}}\right)+d_{2} \cos \left(\sqrt{\phi_{1}}\right)\right\}, D_{5}=\frac{P}{B M^{2}}+D_{3}\left\{d_{3} \sin \left(\sqrt{\phi_{2}}\right)-d_{4} \cos \left(\sqrt{\phi_{2}}\right)\right\} \\
& D_{6}=\frac{P}{M^{2}}-\frac{C P}{B M^{2}}-D_{2} d_{2}+D_{3} d_{4}, D_{7}=\frac{1}{M}\left(\frac{D_{3} d_{3} \sqrt{\phi_{2}}}{m h}-D_{2} d_{1} \sqrt{\phi_{1}}\right), D_{8}=D_{6}-D_{4} e^{M}+D_{5} e^{M \sqrt{B}} \\
& D_{9}=D_{7}+D_{4} e^{M}+k_{1} D_{5} e^{M \sqrt{B}}, D_{10}=D_{8} k_{1}+D_{9}+\left(D_{8} k_{1}-D_{9}\right) e^{2 M \sqrt{B}} \\
& D_{11}=\left(k_{1}+1\right)\left\{1-e^{2 M(1+\sqrt{B})}\right\}+\left(k_{1}-1\right)\left\{e^{2 M \sqrt{B}}-e^{2 M}\right\} \\
& D_{12}=D_{9}\left(1-e^{2 M}\right)-D_{8}\left(1+e^{2 M}\right), \\
& G_{1}=\frac{1}{B M^{2}-\phi_{2}}, G_{2}=\frac{1}{4 B M^{2}-\phi_{2}}, G_{3}=\sqrt{\phi_{2}}+B M^{2} \\
& G_{4}=\sqrt{\phi_{2}}-B M^{2}, G_{5}=\frac{1}{G_{2}^{2}-\phi_{2}}, G_{6}=\frac{1}{G_{3}^{2}-\phi_{2}}, G_{7}=M^{2} G_{2}\left(c_{7}^{2}-c_{8}^{2}\right)(F-B D) / 2 \\
& G_{8}=M^{2} G_{2} c_{7} c_{8}(F+B D), G_{9}=A M^{2} A_{5} G_{5}\left(c_{4} c_{8}-c_{3} c_{7}\right)\left(F-B D \sqrt{\phi_{2}}\right) \\
& G_{10}=A M^{2} A_{5} G_{6}\left(c_{3} c_{7}+c_{4} c_{8}\right)\left(F+B D \sqrt{\phi_{2}}\right), G_{11}=A M^{2} A_{5} G_{5}\left(c_{4} c_{7}+c_{3} c_{8}\right)\left(F+B D \sqrt{\phi_{2}}\right) \\
& G_{12}=A M^{2} A_{5} G_{6}\left(c_{3} c_{8}-c_{4} c_{7}\right)\left(F-B D \sqrt{\phi_{2}}\right), G_{13}=\left(\frac{A^{2} A_{5}^{2} c_{3} c_{4}}{3}\right)\left(\frac{F M^{2}}{\phi_{2}}+D\right) \\
& G_{14}=\left(\frac{A^{2} A_{5}^{2}\left(c_{3}^{2}-c_{4}^{2}\right)}{6}\right)\left(\frac{F M^{2}}{\phi_{2}}-D\right), G_{15}=\frac{2 C P F G_{1} M^{2} c_{7}}{B M^{2}}, G_{16}=\frac{2 C P F G_{1} M^{2} c_{8}}{B M^{2}}, G_{17}=\frac{A C P F M^{2} A_{5} c_{3}}{B M^{2} \sqrt{\phi_{2}}} \\
& G_{18}=\frac{A C P F M^{2} A_{5} c_{4}}{B M^{2} \sqrt{\phi_{2}}}, G_{19}=\frac{C F P^{2}}{B^{2} M^{2} \phi_{2}}+\frac{M^{2}(F+B D)}{2 \phi_{2}}\left(c_{7}^{2}+c_{8}^{2}\right)+\frac{A^{2} A_{5}^{2}}{2}\left(c_{3}^{2}+c_{4}^{2}\right)\left(\frac{F M^{2}}{\phi_{2}}+D\right) \\
& a_{0}=\frac{1}{4 \phi_{1}+M^{2}}, a_{1}=\frac{1}{4 M^{2}+\phi_{1}}, a_{2}=\frac{1}{M^{2}+\phi_{1}}, a_{3}=2 M^{2} a_{1} d_{5}^{2}, a_{4}=2 M^{2} a_{1} d_{6}^{2} \\
& a_{5}=\frac{D_{2}^{2}}{6}\left(d_{1}^{2}-d_{2}^{2}\right)\left(1-\frac{M^{2}}{\phi_{1}}\right), a_{6}=a_{0} d_{5} D_{2}\left(d_{2} M^{2}+2 d_{2} \phi_{1}-M d_{1} \sqrt{\phi_{1}}\right) \\
& a_{7}=a_{0} d_{5} D_{2}\left(d_{1} M^{2}+2 d_{1} \phi_{1}+M d_{2} \sqrt{\phi_{1}}\right), a_{8}=a_{0} d_{6} D_{2}\left(d_{1} M^{2}+2 d_{1} \phi_{1}-M d_{2} \sqrt{\phi_{1}}\right) \\
& a_{9}=a_{0} d_{6} D_{2}\left(d_{2} M^{2}+2 d_{2} \phi_{1}+M d_{1} \sqrt{\phi_{1}}\right), a_{10}=\frac{d_{1} d_{2} D_{2}^{2}}{3}\left(\frac{M^{2}}{\phi_{1}}-1\right), a_{11}=2 P d_{5} a_{2} \\
& a_{12}=2 P d_{6} a_{2}, a_{13}=\frac{P D_{2} d_{1}}{\sqrt{\phi_{1}}}, a_{14}=\frac{P D_{2} d_{2}}{\sqrt{\phi_{1}}}, a_{15}=\frac{P^{2}}{M^{2} \phi_{1}}+\frac{D_{2}^{2}}{2}\left(1+\frac{M^{2}}{\phi_{1}}\right)\left(d_{1}^{2}+d_{2}^{2}\right) \\
& b_{0}=2 B M^{2} D_{3} \sqrt{\phi_{2}}, b_{1}=B M^{2} d_{7}^{2}, b_{2}=B M^{2} d_{8}^{2}, b_{3}=\phi_{2} d_{3}^{2} D_{3}^{2}, b_{4}=\phi_{2} d_{4}^{2} D_{3}^{2} \\
& b_{5}=2 B M^{2} d_{7} d_{8}, b_{6}=b_{0} d_{3} d_{7}, b_{7}=b_{0} d_{4} d_{7}, b_{8}=b_{0} d_{3} d_{8}, b_{9}=b_{0} d_{4} d_{8} \text {, } \\
& b_{10}=d_{3} d_{4} D_{3}^{2} \phi_{2}, c_{1}=c_{3}=\frac{A_{1} \sinh \left(\sqrt{\phi_{2}}\right)}{A_{2}}, c_{2}=\frac{\cosh \left(\sqrt{\phi_{2}}\right)}{A_{2}}, c_{4}=A_{1} \cdot c_{2},
\end{aligned}
$$




$$
\begin{aligned}
& c_{5}=\frac{A_{8} \sinh (M \sqrt{B})+B_{1} A_{9} \sinh (M)}{A_{10}}, c_{6}=A_{7}+B_{1} c_{8}, c_{7}=c_{5}-A_{6}, c_{8}=\frac{A_{8} \cosh (M \sqrt{B})-A_{9} \cosh (M)}{A_{10}} \\
& c_{9}=H_{4}+c_{11}, c_{10}=\frac{H_{5} \cosh \left(\sqrt{\phi_{2}}\right)-H_{6} \cosh \left(\sqrt{\phi_{1}}\right)}{A_{2}}, c_{11}=\frac{H_{5} A_{1} \sinh \left(\sqrt{\phi_{2}}\right)+\sinh \left(\sqrt{\phi_{1}}\right)}{A_{2}} \\
& c_{12}=H_{3}+A_{1} c_{10}, c_{13}=K_{3}+c_{15}, c_{14}=\frac{M \sqrt{B} K_{6} \cosh (M \sqrt{B})-K_{7} \cosh (M)}{K_{5}} \\
& C_{15}=\frac{m h M K_{6} \sinh (M \sqrt{B})-K_{7} \sinh (M)}{K_{5}}, c_{16}=\frac{c_{15} \cosh (M \sqrt{B})-K_{2}}{\sinh (M \sqrt{B})}, k_{1}=\frac{\sqrt{B}}{m h} \\
& d_{1}=\frac{\cos \left(\sqrt{\phi_{2}}\right)}{D_{1}}, d_{2}=d_{4}=\frac{A_{1} \sin \left(\sqrt{\phi_{2}}\right)}{D_{1}}, d_{3}=\frac{A_{1} \cos \left(\sqrt{\phi_{2}}\right)}{D_{1}}, d_{5}=\frac{D_{10}}{D_{11}}, d_{6}=D_{4} e^{M}-d_{5} e^{2 M}, \\
& d_{7}=D_{5} e^{M \sqrt{B}}-d_{8} e^{2 M \sqrt{B}}, d_{8}=\frac{D_{12}}{D_{11}}, d_{9}=\frac{h_{11}}{D_{1}}, d_{10}=h_{3}+d_{12}, d_{11}=A_{1} d_{9}+h_{8}, \\
& d_{12}=\frac{h_{12}}{D_{1}}, d_{13}=\frac{N_{4}}{N_{3}}, d_{14}=k_{2} e^{M}-d_{13} e^{2 M}, d_{15}=k_{3} e^{M \sqrt{B}}-d_{16} e^{2 M \sqrt{B}}, d_{16}=\frac{N_{5}}{N_{3}} \\
& f_{1}=d_{3}^{2} D_{3}^{2}, f_{2}=d_{4}^{2} D_{3}^{2}, f_{3}=\frac{2 C P d_{7}}{B M^{2}}, f_{4}=2 d_{3} d_{7} D_{3}, f_{5}=2 d_{4} d_{7} D_{3}, f_{6}=\frac{2 C P d_{8}}{B M^{2}}, \\
& f_{7}=2 d_{3} d_{8} D_{3}, f_{8}=2 d_{4} d_{8} D_{3} f_{9}=\frac{2 C P d_{3} D_{3}}{B M^{2}}, f_{10}=\frac{2 C P d_{4} D_{3}}{B M^{2}}, f_{11}=d_{3} d_{4} D_{3}^{2}, f_{12}=\frac{C^{2} P^{2}}{B M^{2}}+2 d_{7} d_{8} \\
& g_{1}=\frac{1}{4 B M^{2}+\phi_{2}}, g_{2}=\frac{1}{B M^{2}\left(4 \phi_{2}+B M^{2}\right)}, g_{3}=\frac{1}{B M^{2}+\phi_{2}}, g_{4}=g_{1}\left(D^{2} b_{1}+F M^{2} d_{7}^{2}\right) \\
& g_{5}=g_{1}\left(D^{2} b_{2}-F M^{2} d_{8}^{2}\right), g_{6}=\frac{\left[D^{2}\left(b_{4}-b_{3}\right)+F M^{2}\left(f_{1}-f_{2}\right)\right]}{6 \phi_{2}} \\
& g_{7}=g_{2}\left[\left(2 M b_{6} D^{2}+2 F M^{3} f_{5}\right) \sqrt{B \phi_{2}}-B M^{2} D^{2} b_{7}+B F M^{4} f_{4}\right] \\
& g_{8}=g_{2}\left[B M^{2} D^{2} b_{6}+2 \sqrt{B \phi_{2}}\left(M D^{2} b_{7}-F M^{3} f_{4}\right)-B F M^{4} f_{5}\right] \\
& g_{9}=g_{2}\left[2 \sqrt{B \phi_{2}}\left(M D^{2} b_{9}+F M^{3} f_{7}\right)+B F M^{4} f_{8}-B M^{2} D^{2} b_{8}\right] \\
& g_{10}=g_{2}\left[2 \sqrt{B \phi_{2}}\left(F M^{3} f_{8}-M D^{2} b_{8}\right)-B F M^{4} f_{7}-B M^{2} D^{2} b_{9}\right. \\
& g_{11}=\frac{F M^{2} f_{11}-D^{2} b_{10}}{3 \phi_{2}}, g_{12}=\frac{F M^{2} f_{9}}{2 \sqrt{\phi_{2}}}, g_{13}=\frac{F M^{2} f_{10}}{2 \sqrt{\phi_{2}}}, g_{14}=F M^{2} f_{3} g_{3}, g_{15}=F M^{2} f_{6} g_{3} \\
& g_{16}=\left[D^{2}\left(b_{3}+b_{4}-2 b_{5}\right)+F M^{2}\left(f_{1}+f_{2}+2 f_{12}\right)\right] / 2 \phi_{2} \\
& h_{1}=a_{3} e^{2 M}+a_{4} e^{-2 M}-a_{5} \cos \left(2 \sqrt{\phi_{1}}\right)+\left\{2 a_{6} \cos \left(\sqrt{\phi_{1}}\right)+2 a_{7} \sin \left(\sqrt{\phi_{1}}\right)-a_{11}\right\} e^{M} \\
& +\left[2 a_{8} \sin \left(\sqrt{\phi_{1}}\right)+2 a_{9} \cos \left(\sqrt{\phi_{1}}\right)-a_{12}\right\} e^{-M}-a_{10} \sin \left(2 \sqrt{\phi_{1}}\right)-a_{13} \cos \left(\sqrt{\phi_{1}}\right)+a_{14} \sin \left(\sqrt{\phi_{1}}\right)+a_{15} \\
& h_{2}=g_{4} e^{-2 M \sqrt{B}}-g_{5} e^{2 M \sqrt{B}}+g_{6} \cos \left(2 \sqrt{\phi_{2}}\right)-\left\{g_{7} \sin \left(\sqrt{\phi_{2}}\right)-g_{8} \cos \left(\sqrt{\phi_{2}}\right)+g_{14}\right\} e^{-M \sqrt{B}} \\
& +\left\{g_{9} \cos \left(\sqrt{\phi_{2}}\right)+g_{10} \sin \left(\sqrt{\phi_{2}}\right)-g_{15}\right\} e^{M \sqrt{B}}+g_{11} \sin \left(2 \sqrt{\phi_{2}}\right)-g_{12} \cos \left(\sqrt{\phi_{2}}\right)-g_{13} \sin \left(\sqrt{\phi_{2}}\right)+g_{16} \\
& h_{3}=a_{3}+a_{4}+a_{5}+2 a_{6}+2 a_{9}-a_{11}-a_{12}+a_{15}-g_{4}+g_{5}-g_{6}-g_{8}-g_{9}+g_{14}+g_{15}-g_{16} \\
& h_{4}=2 M a_{3}-2 M a_{4}+2 M a_{6}-2 M a_{9}-M a_{11}+M a_{12}-a_{13}, h_{5}=a_{7}+a_{8}+a_{10} \\
& h_{6}=M \sqrt{B}\left(2 g_{4}+2 g_{5}+g_{8}-g_{9}-g_{14}+g_{15}\right)+g_{12}, h_{7}=-g_{7}+g_{10}+2 g_{11} \\
& h_{8}=\left(\frac{k h h_{4}+h_{6}}{\sqrt{\phi_{2}}}\right)+2 A_{1} h_{5}+h_{7}, h_{9}=h_{2}-h_{8} \sin \left(\sqrt{\phi_{2}}\right), h_{10}=h_{1}-h_{3} \cos \left(\sqrt{\phi_{1}}\right) \\
& h_{11}=h_{9} \cos \left(\sqrt{\phi_{1}}\right)+h_{10} \cos \left(\sqrt{\phi_{2}}\right), h_{12}=h_{9} \sin \left(\sqrt{\phi_{1}}\right)+h_{10} \mathrm{~A}_{1} \sin \left(\sqrt{\phi_{2}}\right) \\
& l_{1}=\frac{1}{\phi_{1}+2 M \sqrt{\phi_{1}}}, l_{2}=\frac{1}{\phi_{1}-2 M \sqrt{\phi_{1}}}, l_{3}=\frac{1}{4 \phi_{1}-M^{2}}, l_{4}=A_{3} B_{1} c_{9}, l_{5}=A_{3} B_{1} c_{10}, l_{6}=A_{3} l_{3} B_{7} \\
& l_{7}=\frac{A_{3} B_{8}}{3 M^{2}}, l_{8}=\frac{A_{3} B_{9}}{2 M}, l_{9}=\frac{A_{3} B_{10}}{2 M}, l_{10}=A_{3} l_{1} B_{11}, l_{11}=A_{3} l_{2} B_{12}, l_{12}=A_{3} l_{1} B_{13}, l_{13}=A_{3} l_{2} B_{14}, l_{14}=A_{3} B_{1} B_{15},
\end{aligned}
$$




$$
\begin{aligned}
& l_{15}=2 \sqrt{\phi} A_{3} B_{1}^{2} B_{15}, l_{16}=A_{3} l_{3} B_{16}, l_{17}=A_{3} B_{1} B_{17}, l_{18}=2 \sqrt{\phi} A_{3} B_{1}^{2} B_{17}, l_{19}=\frac{A_{3} B_{18}}{M^{2}}, \\
& m_{1}=\frac{1}{\phi_{2}+2 M \sqrt{B \phi_{2}}}, m_{2}=\frac{1}{\phi_{2}-2 M \sqrt{B \phi_{2}}}, m_{3}=\frac{1}{4 \phi_{1}-B M^{2}} \\
& m_{4}=A_{3} A G_{1} c_{11}, m_{5}=A_{3} A G_{1} c_{12}, m_{6}=\frac{A A_{3} G_{8}}{3 B M^{2}}, m_{7}=\frac{A A_{3} G_{8}}{3 B M^{2}}, m_{8}=A A_{3} m_{1} G_{9} \\
& m_{9}=A A_{3} m_{2} G_{10}, m_{10}=A A_{3} m_{1} G_{11}, m_{11}=A A_{3} m_{2} G_{12}, m_{12}=A A_{3} m_{3} G_{13} \\
& m_{13}=A A_{3} m_{3} G_{14}, m_{14}=\frac{A A_{3} G_{15}}{2 M \sqrt{B}}, m_{15}=\frac{A A_{3} G_{16}}{2 M \sqrt{B}}, m_{16}=A A_{3} G_{1} G_{17} \\
& m_{17}=2 \sqrt{\phi_{2}} A A_{3} G_{1}{ }^{2} G_{17}, m_{18}=A A_{3} G_{1} G_{18}, m_{19}=2 \sqrt{\phi_{2}} A A_{3} G_{1}{ }^{2} G_{18}, m_{20}=\frac{A A_{3} G_{19}}{B M^{2}} \\
& n_{0}=\frac{2 M A_{3}}{\sqrt{\phi_{1}}}, n_{1}=A_{3} d_{9} a_{2}, n_{2}=A_{3} d_{10} a_{2}, n_{3}=\frac{A_{3} a_{3}}{3 M^{2}}, n_{4}=\frac{A_{3} a_{4}}{3 M^{2}}, \\
& n_{5}=A_{3} a_{5} a_{0}, n_{6}=A_{3} a_{10} a_{0}, n_{7}=\frac{A_{3} a_{11}}{2 M}, n_{8}=\frac{A_{3} a_{12}}{2 M}, n_{9}=2 a_{1}\left(n_{0} a_{6}-A_{3} a_{7}\right), \\
& n_{10}=2 a_{1}\left(n_{0} a_{7}+A_{3} a_{6}\right), n_{11}=2 a_{1}\left(n_{0} a_{9}+A_{3} a_{8}\right), n_{12}=2 a_{1}\left(n_{0} a_{8}-A_{3} a_{9}\right) \\
& n_{13}=A_{3} a_{13} a_{2}, n_{14}=2 \sqrt{\phi_{1}} A_{3} a_{13} a_{2}^{2}, n_{15}=A_{3} a_{14} a_{2}, n_{16}=2 \sqrt{\phi_{1}} A_{3} a_{14} a_{2}^{2}, n_{17}=\frac{A_{3} a_{15}}{M^{2}} \\
& p_{0}=\frac{1}{4 \phi_{2}+B M^{2}}, p_{1}=A A_{3} d_{11} g_{3}, p_{2}=A A_{3} d_{12} g_{3}, p_{3}=\frac{A A_{3} g_{4}}{3 B M^{2}}, p_{4}=\frac{A A_{3} g_{5}}{3 B M^{2}}, \\
& p_{5}=A A_{3} g_{6} g_{0} p_{6}=A A_{3} g_{11} g_{0}, p_{7}=\frac{A A_{3} g_{14}}{2 M \sqrt{B}}, p_{8}=\frac{A A_{3} g_{15}}{2 M \sqrt{B}}, p_{9}=g_{1}\left(n_{0} A \sqrt{B} g_{8}-A A_{3} g_{7}\right) \\
& p_{10}=g_{1}\left(n_{0} A \sqrt{B} g_{7}+A A_{3} g_{8}\right), p_{11}=g_{1}\left(n_{0} A \sqrt{B} g_{9}-A A_{3} g_{10}\right) \\
& p_{12}=g_{1}\left(n_{0} A \sqrt{B} g_{10}+A A_{3} g_{9}\right), p_{13}=A A_{3} g_{12} g_{3}, p_{14}=2 A A_{3} g_{3}^{2} g_{12} \sqrt{\phi_{2}}, p_{15}=A A_{3} g_{13} g_{3} \\
& p_{16}=2 A A_{3} g_{3}^{2} g_{13} \sqrt{\phi_{2}}, p_{17}=A A_{3} g_{16} / B M^{2} \\
& k_{2}=-n_{1} \sin \left(\sqrt{\phi_{1}}\right)-n_{2} \cos \left(\sqrt{\phi_{1}}\right)-n_{3} e^{2 M}-n_{4} e^{-2 M}-n_{5} \cos \left(2 \sqrt{\phi_{1}}\right)-n_{6} \sin \left(2 \sqrt{\phi_{1}}\right)+n_{7} e^{M}-n_{8} e^{-M} \\
& -n_{9} e^{M} \sin \left(\sqrt{\phi_{1}}\right)+n_{10} e^{M} \cos \left(\sqrt{\phi_{1}}\right)+n_{11} e^{-M} \sin \left(\sqrt{\phi_{1}}\right)-n_{12} e^{-M} \cos \left(\sqrt{\phi_{1}}\right)-n_{13} \cos \left(\sqrt{\phi_{1}}\right) \\
& -n_{14} \sin \left(\sqrt{\phi_{1}}\right)+n_{15} \sin \left(\sqrt{\phi_{1}}\right)+n_{16} \cos \left(\sqrt{\phi_{1}}\right)+n_{17} \\
& k_{3}=p_{1} \sin \left(\sqrt{\phi_{2}}\right)-p_{2} \cos \left(\sqrt{\phi_{2}}\right)-p_{3} e^{-2 M \sqrt{B}}+p_{4} e^{2 M \sqrt{B}}+p_{5} \cos \left(2 \sqrt{\phi_{2}}\right)+p_{6} \sin \left(2 \sqrt{\phi_{2}}\right) \\
& -b_{7} e^{-M \sqrt{B}}+p_{8} e^{M \sqrt{B}}+p_{9} e^{-M \sqrt{B}} \sin \left(\sqrt{\phi_{2}}\right)+p_{10} e^{-M \sqrt{B}} \cos \left(\sqrt{\phi_{2}}\right)-p_{11} e^{M \sqrt{B}} \sin \left(\sqrt{\phi_{2}}\right) \\
& +p_{12} e^{M \sqrt{B}} \cos \left(\sqrt{\phi_{2}}\right)-p_{13} \cos \left(\sqrt{\phi_{2}}\right)+p_{14} \sin \left(\sqrt{\phi_{2}} y\right)-p_{15} \sin \left(\sqrt{\phi_{2}}\right)-p_{16} \cos \left(\sqrt{\phi_{2}}\right)+p_{17} \\
& k_{4}=p_{2}+p_{3}-p_{4}-p_{5}-p_{10}-p_{12}+p_{16}-p_{17}-n_{2}-n_{3}-n_{4}-n_{5}+n_{10}-n_{12}+n_{16}+n_{17} \\
& k_{5}=\frac{1}{m M h}, k_{6}=\frac{\sqrt{\phi_{2}}}{M}, k_{7}=k_{5} \sqrt{\phi_{2}}\left(p_{1}+2 p_{6}+p_{9}-p_{11}+p_{14}\right) \text {, } \\
& k_{8}=k_{1}\left(2 p_{3}+2 p_{4}-p_{10}+p_{12}\right), k_{9}=k_{5}\left(p_{8}-p_{7}-p_{13}\right), \\
& k_{10}=k_{6}\left(n_{1}+2 n_{6}+n_{9}-n_{11}-n_{14}\right), k_{11}=n_{8}-n_{7}+n_{13}, k_{12}=2 n_{3}-2 n_{4}-n_{10}-n_{12} \\
& k_{13}=k_{7}+k_{8}+k_{9}-k_{10}-k_{12}-\left(k_{11} / M\right) \\
& N_{1}=k_{4}+k_{3} e^{M \sqrt{B}}-k_{2} e^{M}, N_{2}=k_{13}+k_{1} k_{3} e^{M \sqrt{B}}+k_{2} e^{M}, \\
& N_{3}=k_{1}\left(1-e^{2 M}\right)\left(1+e^{2 \sqrt{B} M}\right)+\left(1+e^{2 M}\right)\left(1-e^{2 \sqrt{B} M}\right) \text {, } \\
& N_{4}=N_{1} k_{1}\left(1+e^{2 \sqrt{B} M}\right)+N_{2}\left(1+e^{2 \sqrt{B} M}\right), N_{5}=N_{2}\left(1-e^{2 M}\right)-N_{1}\left(1+e^{2 M}\right)
\end{aligned}
$$




$$
\begin{aligned}
& H_{1}=B_{7} \cosh \left(2 \sqrt{\phi_{1}}\right)+B_{16} \sinh \left(2 \sqrt{\phi_{1}} y\right)+B_{8} \sinh (2 M)+B_{9} \cosh (M)-B_{10} \sinh (M)-B_{11} \sinh \left(B_{3}\right)-B_{12} \sinh \left(B_{4}\right) \\
& -B_{13} \cosh \left(B_{3}\right)-B_{14} \cosh \left(B_{4}\right)-B_{15} \cosh \left(\sqrt{\phi_{1}}\right)+B_{17} \sinh \left(\sqrt{\phi_{1}} y\right)-B_{18} \\
& \mathrm{H}_{2}=G_{7} \cosh (2 M \sqrt{B})-G_{8} \sinh (2 M \sqrt{B})+G_{9} \cosh \left(G_{3}\right)-G_{10} \cosh \left(G_{4}\right)+G_{11} \sinh \left(G_{3}\right)-G_{12} \sinh \left(G_{4}\right)-G_{13} \sinh \left(2 \sqrt{\phi_{1}}\right) \\
& +G_{14} \cosh \left(2 \sqrt{\phi_{2}}\right)-\mathrm{G}_{15} \cosh (M \sqrt{B})+\mathrm{G}_{16} \sinh (M \sqrt{B})+G_{17} \sinh \left(\sqrt{\phi_{2}}\right)+G_{18} \cosh \left(\sqrt{\phi_{2}}\right)-G_{19} \\
& H_{3}=\frac{K H}{\sqrt{\phi_{1}}}\left(2 M B_{8}-M B_{10}-B_{3} B_{11}-B_{4} B_{12}-B_{15}\right)-\sqrt{\phi_{2}}\left(2 M G_{8} \sqrt{B}-G_{3} G_{11}+G_{4} G_{12}-M G_{16} \sqrt{B}-G_{18}\right)+2 A_{1} F_{16}-2 G_{13} \\
& H_{4}=B_{7}+B_{9}-B_{13}-B_{14}-B_{18}-G_{7}-G_{9}+G_{10}-G_{14}+G_{15}+G_{18} \\
& H_{5}=H_{1}-H_{4} \cosh \left(\sqrt{\phi_{1}}\right) \\
& H_{6}=H_{2}-H_{3} \sinh \left(\sqrt{\phi_{2}}\right) \\
& K_{1}=-\left(l_{4}+l_{14}-l_{18}\right) \cosh \left(\sqrt{\varphi_{1}}\right)-\left(l_{5}+l_{15}-l_{17}\right) \sinh \left(\sqrt{\varphi_{1}}\right)-l_{6} \cosh \left(2 \sqrt{\varphi_{1}}\right)-l_{7} \sinh (2 M)-l_{8} \sinh (M) \\
& -l_{9} \cosh (M)+l_{10} \sinh \left(B_{3}\right)+l_{11} \sinh \left(B_{4}\right)+l_{12} \cosh \left(B_{3}\right)+l_{13} \cosh \left(B_{4}\right)-l_{16} \sinh \left(2 \sqrt{\varphi_{1}}\right)-l_{19} \\
& K_{2}=-\left(m_{4}-m_{17}-m_{18}\right) \cosh \left(\sqrt{\varphi_{2}}\right)+\left(m_{5}+m_{16}+m_{19}\right) \sinh \left(\sqrt{\varphi_{2}}\right)-m_{6} \cosh (2 M \sqrt{B})+m_{7} \sinh (2 M \sqrt{B}) \\
& -m_{8} \cosh \left(G_{3}\right)+m_{9} \cosh \left(G_{4}\right)-m_{10} \sinh \left(G_{3}\right)+m_{11} \sinh \left(G_{4}\right)+m_{12} \sinh \left(2 \sqrt{\varphi_{2}}\right) \\
& -m_{13} \cosh \left(2 \sqrt{\varphi_{2}}\right)+m_{14} \sinh (M \sqrt{B})+m_{15} \cosh (M \sqrt{B})-m_{20} \\
& K_{3}=-l_{4}-l_{6}+l_{12}+l_{13}+l_{18}-l_{19}+m_{4}+m_{6}+m_{8}-m_{9}+m_{13}-m_{17}+m_{20} \\
& K_{4}=l_{9}-l_{14}-2 M l_{7}+B_{4} l_{11}+B_{3} l_{10}-\sqrt{\varphi_{1}}\left(l_{5}+l_{15}+2 l_{16}\right)+\left(\frac{1}{m h}\right)\left\{m_{15}+m_{18}+2 M \sqrt{B} m_{7}-m_{10} G_{3}+m_{11} G_{4}\right. \\
& \left.+\sqrt{\varphi_{2}}\left(m_{5}+m_{9}+2 m_{12}\right)\right\} \\
& K_{5}=(m h M) \cosh (M) \sinh (M \sqrt{B})+(M \sqrt{B}) \sinh (M) \cosh (M \sqrt{B}) \\
& K_{6}=K_{1}-K_{3} \cosh (M) \\
& K_{7}=M \sqrt{B} K_{2}-m h K_{4} \sinh (M \sqrt{B})
\end{aligned}
$$

\section{References}

[1] Hartmann, J., 1937, "Hg-dynamics I Theory of the Laminar Flow of an Electrically Conductive Liquid in a Homogeneous Magnetic Field," kgl Danskevidenskab Selkab Mat Fys Medd., 15.

[2] Seigel, R., 1958, "Effect of Magnetic Field on Forced convective Heat transfer in a Parallel Plate Channel," J Appl. Mech. 25, 415.

[3] Osterle, J. F., Young, F. J., 1961, "Natural Convection between Heated Vertical Plates in a Horizontal Magnetic Field," J. Fluid Mech. 11, pp. 512-518.

[4] Perlmutter, M., and Seigal, R., 1961, "Heat Transfer to an Electrically Conducting Fluid Flowing in a Channel with Transverse Magnetic Field," NACA TN D-875.

[5] Romig, M. F., 1961, "The Influence of Electric and Magnetic Fields on Heat Transfer to Electrically Conducting Fluids," Adv. Heat Transfer vol 1, New York, Academic Press.

[6] Umavathi, J. C., 1996, "A Note on Magnetoconvection in a Vertical Enclosure,” Int. J. Nonlinear Mechanics 31, pp. 371376.

[7] Thome, R. J., 1964, Effect of Transverse Magnetic Field on
Vertical Two Phase Flow through a Rectangular Channel, Argonne National Laboratory Report No. ANL 6854.

[8] Lohrasbi, J., and Sahai, V., 1988, "Magnetohydrodynamic Heat Transfer in Two Phases Flow between Parallel Plates," Appl. Sci. Res. 45, pp. 53-66.

[9] Malashetty, M. S., and Leela, V., 1991, "Magnetohydrodynamic Heat Transfer in Two Fluid Flow," Proceeding of the National Heat Transfer, Conf. AIChE \&ASME, HTD, p. 159.

[10] Malashetty, M. S., and Leela, V., 1992, "Magnetohydrodynamic Heat Transfer in Two Phase Flow," Int. J Eng. Sci., 30, pp. 371-377.

[11] Chamkah, A. J., 2000, "Flow of Two Immiscible Fluid in Porous and Nonporous Channels," J. Fluid Eng. 122, pp. 117124.

[12] Prakash, S., 1970, "Liquid Flowing Down an Open Inclined Channel," Indian J. Pure Appl. Math., 2, pp. 1093-109.

[13] Verma, P. D., and Vyas, H. K., 1980, "Viscous Flow Down an Open Inclined Channel with Naturally Permeability Bed," Indian J. Pure Appl. Math. 11, pp.165-172.

[14] Malashetty, M. S., and. Umavathi, J. C., 1997, “Two-Phase Magnetohydrodynamic Flow and Heat Transfer in an Inclined Channel," Int. J. of Multiphase Flow, 23, pp. 545-560. 
[15] Malashetty, M. S., Umavathi, J. C., and Prathap, Kumar, J., 2001, "Convective Magnetohydrodynamic Two Fluid Flow and Heat Transfer in an Inclined Channel," Heat and Mass Transfer, 37, pp. 259-264.

[16] Wang, X., and Robillard, L., 1995, "Mixed Convection in an Inclined Channel with Localized Heat Sources," Numerical Heat Transfer-Part-A: Application, 28, pp.355-373.

[17] Umavathi, J. C., Chamkah, A. J., and Shekar, M., 2014, "Flow and Heat Transfer of Two Micropolar Fluids Separated by a Viscous Fluid Layer," International Journal of Microscale and Nanoscale Thermal and Fluid Transport Phenomena, 5, pp. 23-49.

[18] Chamkah, A. J., Mansour, M. A., and Ahmad, S. E., 2010, "Double-Diffusive Natural Convection in Inclined Finned Triangular Porous Enclosures in the Presence of Heat Generation/Absorption Effects." Heat and Mass Transfer, 46, pp. 757-768. 